\title{
Architecting a Hardware-Managed Hybrid DIMM Optimized for Cost/Performance
}

\author{
Fred Ware ${ }^{1}$, Javier Bueno ${ }^{2}$, Liji Gopalakrishnan ${ }^{1}$, Brent Haukness ${ }^{1}$, Chris Haywood ${ }^{1}$, Toni Juan ${ }^{2}$, Eric Linstadt ${ }^{1}$, Sally A. \\ McKee $^{3}$, Steven C. Woo ${ }^{1}$, Kenneth L. Wright ${ }^{1}$, Craig Hampel ${ }^{1}$, Gary Bronner ${ }^{1}$ \\ ${ }^{1}$ Rambus Inc. Sunnyvale, California $\quad{ }^{2}$ Metempsy, Barcelona, Spain $\quad{ }^{3}$ Clemson University, South Carolina \\ \{ware, lijig, haukness, chaywood, elinstadt, swoo, kwright, champel, gbronner\}@rambus.com \\ \{javier.bueno, toni.juan\}@metempsy.com_samckee@clemson.edu
}

\begin{abstract}
Rapidly evolving workloads and exploding data volumes place great pressure on data-center compute, IO, and memory performance, and especially on memory capacity. Increasing memory capacity requires a commensurate reduction in memory cost per bit. DRAM technology scaling has been steadily delivering affordable capacity increases, but DRAM scaling is rapidly reaching physical limits. Other technologies such as Flash, enhanced Flash, Phase Change Memory, and Spin Torque Transfer Magnetic RAM hold promise for creating high capacity memories at lower cost per bit. However, these technologies have attributes that require careful management.

We propose a hybrid DIMM architecture that uses a hardwaremanaged DRAM in front of enhanced Flash, which has much lower read latencies than conventional Flash. We explore the design space of such SCM devices in the context of different technology parameters, evaluating performance and endurance for data-center workloads. Our hybrid memory architecture is commercially realizable and can use standard DIMM form factors, giving it a low barrier to market entry. We find that for workloads like media streaming, enhanced Flash can be combined with DRAM to enable $88 \%$ of the performance of a DRAM-only system of the same capacity at $23 \%$ of the cost, even when factoring in replacement costs due to wear-out. The bottom line is that cost per performance is a factor of 3.8 better than DRAM.
\end{abstract}

\section{INTRODUCTION}

Data-center servers struggle to keep up with rapidly evolving workloads and exploding data volumes. For many Big Data workloads, DRAM capacity is as important as compute, IO, and

Permission to make digital or hard copies of part or all of this work for personal or classroom use is granted without fee provided that copies are not made or distributed for profit or commercial advantage and that copies bear this notice and the full citation on the first page. Copyrights for third-party components of this work must be honored. For all other uses, contact the Owner/Author.

MEMSYS, October 1-4, 2018, Old Town Alexandria, VA, USA

(c) 2018 Copyright is held by the owner/author(s).

ACM ISBN 978-1-4503-6475-1/18/10.

https://doi.org/10.1145/3240302.3240303 memory performance. Increasing capacity in a commercially viable way requires reducing cost per bit. DRAM technology scaling has been delivering steady decreases, but DRAM's scaling ability is reaching physical limits [1]. Fortunately, other Storage Class Memory (SCM) technologies hold promise for creating high capacity memories at low cost per bit. These include enhanced Flash with much lower read latencies, Phase Change Memory (PCM), Resistive RAM (ReRAM), and Spin-Transfer Torque Magnetic RAM (STT-MRAM). However, these technologies have attributes that require careful management to provide high reliability, availability, longevity, and performance.

In particular, these technologies are at least an order of magnitude slower than DRAM, which directly affects response times for datacenter workloads. The challenge, then, is how to leverage SCM to increase capacity and lower cost per bit while delivering acceptable performance.

To that end, we propose a hybrid DIMM architecture that uses a hardware-managed DRAM in front of enhanced Flash. We explore the design space of high-capacity, low cost per bit systems deploying such devices, evaluating performance and endurance for data-center workloads in the context of different technology parameters.

SCM is usually also non-volatile, but in this work we don't take advantage of that aspect of the technology. Likewise, we do not address power and reliability or the susceptibility to/effects of electrical or thermal noise in any of our SCM technologies; such studies are relegated to future work. We make the following contributions:

- We study standard DRAM and enhanced Flash [2, 3, 4] components, and we propose a novel way in which to combine them in a single, hybrid DIMM;

- We use the DRAM as a large cache in front of the enhanced Flash, finding that this eliminates $34-81 \%$ of the total Flash writes on the device for the data-center workloads we study;

- We find that a $16: 1$ enhanced Flash to DRAM capacity ratio with a $4 \mathrm{~KB}$ fetch size performs well; and 
- We find that for some workloads, a memory system based on our architecture can deliver up to $88 \%$ of the performance of a same-capacity DRAM-only system at $23 \%$ of the cost.

Our hybrid memory architecture is commercially realizable and can use standard DIMM form factors. Furthermore, our hardware-managed architecture requires no software changes and minimal hardware changes. All these factors give it a low barrier to market entry. With it, we can build very high-capacity, high-availability main memory systems with good performance per dollar over system lifetime.

\section{BACKGROUND}

In order to better compare technologies, Table 1 presents approximate characteristics for DRAM, Flash, and the most common Storage Class Memory types. Since our hybrid DIMM uses DRAM and NAND Flash, we briefly describe their properties and uses.

DRAM. DRAM has the highest read and write bandwidths, lowest latency, and highest endurance. In server systems, the granularity of last-level cache (LLC) cache lines is typically 64B. A memory configuration that supports $64 \mathrm{~B}$ cache lines can easily support higher cache line sizes such as $128 \mathrm{~B}$ using back-to-back column accesses to DRAM.

DRAM accesses are destructive, requiring a write-back operation. DRAM cells have a retention time of about $100 \mathrm{~ms}$ at normal operating temperatures, requiring periodic refresh operations that expend power without accomplishing useful accesses. This volatility is not a serious drawback and would not itself drive a search for a replacement technology. The real problem with DRAM is its inability to scale as aggressively as it has been. DRAM capacity and associated cost per bit have improved by about six orders of magnitude over DRAM's fivedecade reign as the primary main-memory component, making the technology difficult to replace. Further scaling will be much harder, which means that cost per bit will improve only modestly in the future.

In contrast, SCM tends to be easier to scale with lithographic improvements. Capacity and cost per bit of SCM components are thus expected to improve much more/faster than DRAM as shown in Table 1. Future memory systems will likely incorporate SCM components to increase capacity and reduce cost.

Table 1: Comparison of Memory Technologies

\begin{tabular}{lllllll}
\hline Feature & DRAM & STT -MRAM & ReRAM & PCM & \multicolumn{2}{c}{$\begin{array}{l}\text { Enhanced } \\
\text { Flash }\end{array}$} \\
\hline Relative Cost/bit & 1 & $\sim 1$ & $\sim 0.25$ & $\sim 0.25$ & 0.17 & 0.06 \\
Read access & $30 \mathrm{~ns}$ & $100 \mathrm{~ns}$ & $100 \mathrm{~ns}$ & $300 \mathrm{~ns}$ & $3 \mu \mathrm{s}$ & $45 \mu \mathrm{s}$ \\
Write access & $30 \mathrm{~ns}$ & $100 \mathrm{~ns}$ & $1 \mu \mathrm{s}$ & $300 \mathrm{~ns}$ & $100 \mu \mathrm{s}$ & $660 \mu \mathrm{s}$ \\
Byte-addressable & Yes & Yes & Yes & Yes & No (page) & No (page) \\
Endurance (cycles) & $>10^{16}$ & $\sim 10^{10}$ & $\sim 10^{9}$ & $\sim 10^{9}$ & $\sim 10^{4}$ & $\sim 10^{4}$ \\
Manufacturing volume & High & Low & Low & Low & Medium & High \\
\hline
\end{tabular}

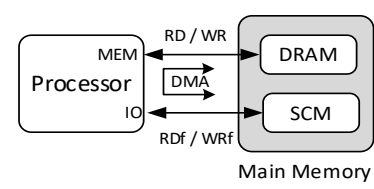

(A)

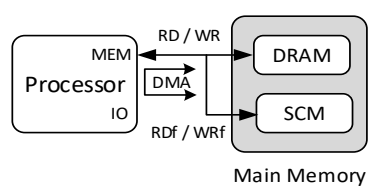

(B)

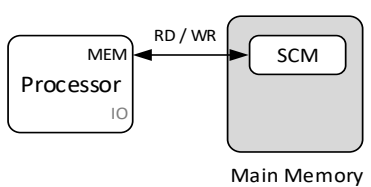

(C)

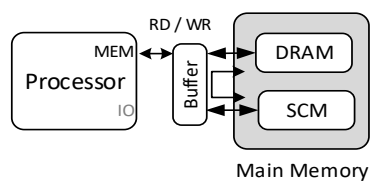

(D)

Figure 1: Example main memory configurations. (A) SCM on IO bus (B) SCM+ DRAM on MEM bus (C) SCM only (D) DRAM as $\mathrm{L} 4$ cache

Flash. Flash technology has the highest latencies and lowest bandwidths in Table 1. It also has the lowest endurance. Flash is not byte-addressable and must be read and written (programmed) in large chunks referred to as pages. It must be prepared for a program operation by erasing even larger blocks. Conventional NAND Flash has thus been used primarily as tertiary storage in the form of SSDs (Solid State Drives). Input/output (IO) operations to block devices typically move large chunks of data, so the larger access granularity and higher latencies are not problematic. This mature technology is in high-volume production, and the smaller cell size, the ability to store multiple bits per cell, and cost- effective 3D stacking make its cost per bit much lower than DRAM.

We use the term enhanced Flash to refer to NAND devices with access latencies $[2,3,4]$ that are an order of magnitude less than conventional Flash (which we do not consider an SCM). Table 2 shows a range of conventional and enhanced Flash devices. The die costs in the rightmost column are relative to the $21 \mathrm{~nm} 8 \mathrm{~Gb}$ DDR4 DRAM die [5] shown in row 5. A present-day 256Gb 3bits/cell V-NAND Flash device [2] is shown in row 1. By operating such a device in a two bit/cell mode, as shown in row 2 , we estimate that read latencies as short as $5 \mu$ should be 
attainable, with a bit density of $2 / 3$ that of the reference NAND device. In a one bit/cell mode, shown in row 3, read latencies as short as $3 \mu$ s are attainable, with a bit density one third of the reference NAND device [6]. With further re-architecting of the NAND die to use a smaller mat size, shown in row 4, we estimate read latencies of $1 \mu \mathrm{s}$ to be attainable at a slight reduction in bit density. In summary, since the read/write latencies of Flash are orders of magnitude worse compared to DRAM, in order to take advantage of the improved bit density and low die cost with minimum impact to the overall execution time, we need smart hybrid memory management techniques.

Table 2: Die Cost and Latency Estimates for Flash Devices

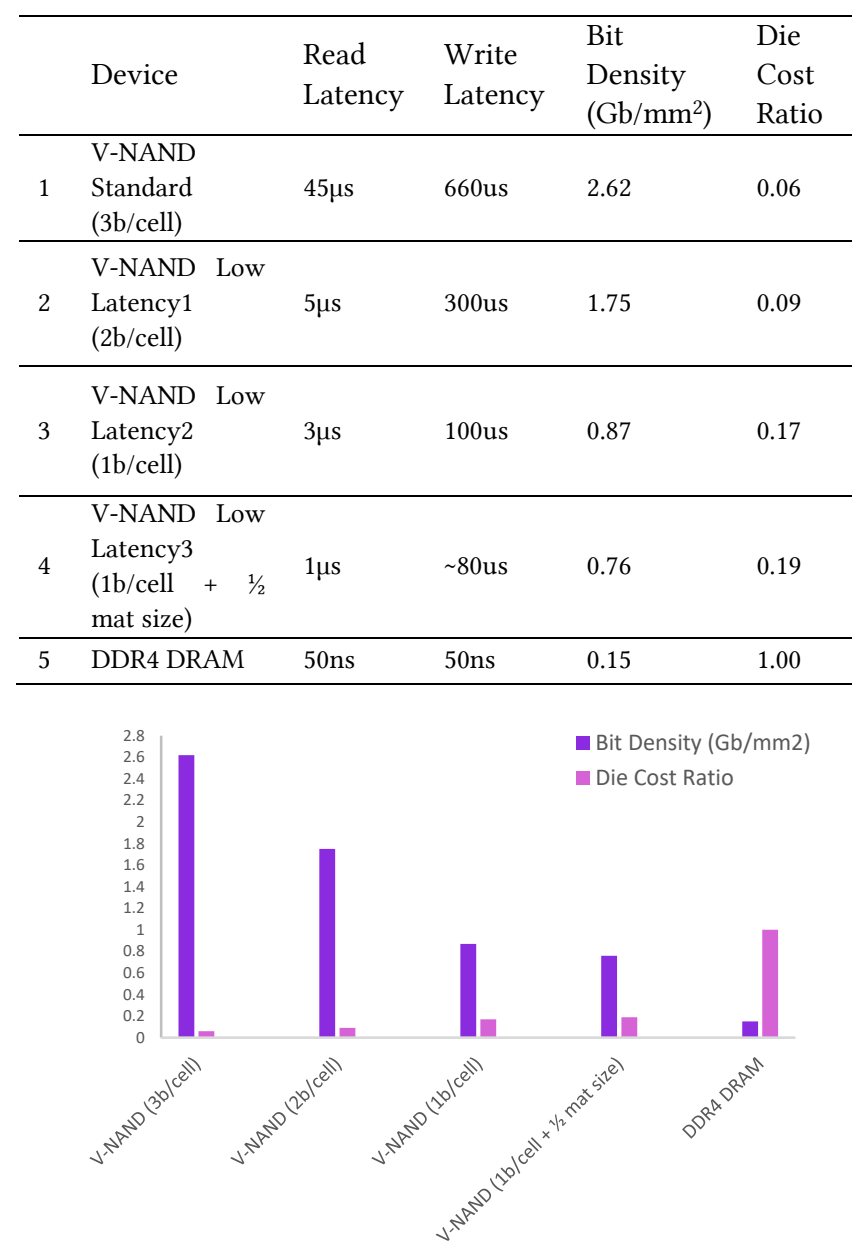

Figure 2: Bit Density and Die Cost Comparison for various Flash Devices compared to DRAM. Die cost goes up as bit density reduces

\section{BUILDING SCM MAIN MEMORIES}

SCM components can be incorporated into the memory hierarchy in several ways, each of which has different costs and benefits. To highlight some of the tradeoffs and frame our discussion, Fig. 1 shows four example configurations.

The processor's on-chip cache hierarchy satisfies most instruction fetch and data load/store operations. When the lastlevel cache (LLC) misses, it fetches a 64B cache-line from physical memory, possibly evicting a cached line to make space and writing it to physical memory if dirty.

A high-performance memory controller generates individual read (RD) and write (WR) operations. It can perform concurrent 64B transactions on the memory (MEM) bus. The memory controller includes control registers for dividing the physical address space into DRAM regions and memory-mapped IO regions. These memory-mapped regions typically reside on the IO bus, which connects via an IO controller.

The LLC can also generate read (RDf) and write (WRf) operations to memory-mapped regions. The IO controller handles these operations and non-cached loads/stores, and it can also perform autonomous Direct Memory Access (DMA) transfers between the MEM and IO buses. The latter bus typically has higher latency and lower bandwidth. We assume the LLC is an L3 cache in the following discussion.

SCM attached to IO bus. Configuration A of Fig. 1 connects DRAM components to the MEM bus and accessed with RD/WR operations from the L3. SCM components are connected to the IO bus and accessed directly with 64-byte RDf/WRf operations from the L3. A software driver sets up DMA transfers between SCM and DRAM. Larger transfer sizes amortize the software overheads better. Configuration A is easy to build, e.g., by placing the SCM components on a PCle card. The software overhead and the latency of going over the IO bus are disadvantages of this organization. Once the data block is in DRAM, software uses load/store instructions to access locations within it.

Configuration $\mathrm{A}$ is similar to a traditional organization in which an SSD (with Flash) is connected to the IO bus as storage or swap space. The OS paging mechanism brings the blocks or pages from SCM to DRAM on page faults.

SCM and DRAM on MEM Bus. Configuration B of Fig. 1 connects both DRAM and SCM components to the MEM bus. The LLC accesses both physical memory regions at a 64-byte granularity, using $\mathrm{RD} / \mathrm{WR}$ operations for the DRAM region and RDf/WRf operations for the SCM region.

When components with different latencies share the MEM bus, the memory controller can pipeline transactions by making an SCM read a two-step operation [7]. A command/address is first sent to fetch data into a buffer on the module. The second command transports the SCM read data using DRAM timings. An SCM write remains a single-step operation using the same timing as for DRAM: the data are buffered on the module, where the slow write access occurs in the background. SCM access operations require status feedback to the memory controller to signal a buffer overflow, a non-deterministic access time, a resource conflict, or any other unpredictable situation. This status could be implemented as a single pipelined bit per MEM transaction to request retry or as part of a standard interface such as NVDIMM$\mathrm{P}$ [8], which is currently under definition. 
If software accesses the SCM directly via load/store instructions in the application software, the memory controller must indicate the longer completion time to the L3. Alternatively, application software can be modified to be aware of this longer access latency, as in Cui et al. [7]. For DMA transfer, the memory controller must perform a series of 64-byte access operations and buffer the data while supporting concurrent RD/WR operations from the L3.

SCM on MEM Bus. Configuration C of Fig. 1 populates the physical memory with only SCM components on the MEM bus. SCM components are directly accessed with 64B RD/WR operations from the LLC, and the memory-mapping of Configurations A and B is therefore not needed. Applications use load/store instructions to access the SCM, albeit with longer latency. Using all the MEM bus bandwidth requires operating multiple SCM components in parallel.

L4 DRAM Cache with SCM backing Memory. This paper focuses on Configuration D of Fig. 1. The DRAMs connect to the MEM bus through a buffer component and are accessed with $\mathrm{RD} / \mathrm{WR}$ operations from the LLC. DRAM thus serves as an L4 cache for the SCM: when a cache miss occurs, this DRAM buffer component informs the memory controller of the longer latency and gets the requested line from SCM. SCM components use the physical address space normally occupied by DRAM. The memory controller must handle the status feedback but needs no major changes.

Configuration D avoids the adoption barriers of the other organizations by requiring no software modification and limited memory controller changes. It can also deliver very good performance while taking advantage of Flash, the slowest, cheapest and most mature component from Table 1. Integrating the SCM and DRAM components on a standard DIMM further lowers the barrier to market entry. Fig. 3 shows the organization of such a DIMM.

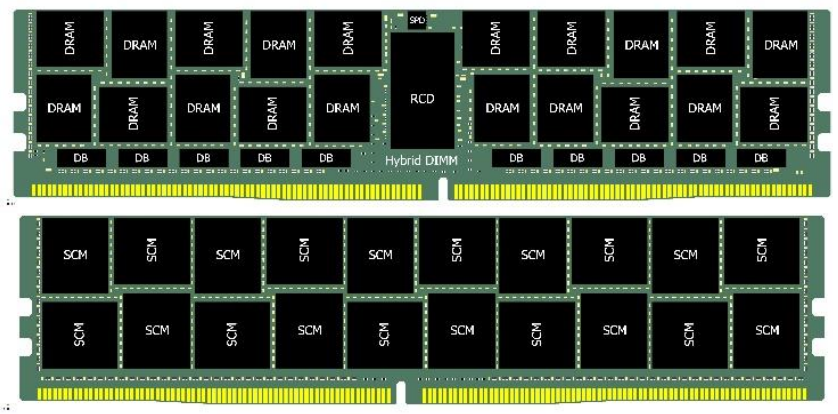

Figure 3: Physical Implementation of Configuration D on a standard DIMM form factor. Front side w/ DRAM and buffer components above; back side w/ SCM components below

\section{ARCHITECTURE}

Fig. 4 shows the logical block diagram of an example implementation of Configuration D. For clarity, we show only half the DIMM module. Five-byte-wide data buffers (DBs) and the command buffer (register clock driver, or RCD) connect to $10 \times 4$ DRAMs and 10 SCM devices. Assuming dual die DRAM packages with $16 \mathrm{~Gb} /$ die and 4 -die-stack Flash packages with $128 \mathrm{~Gb} /$ die, SCM capacity is $16 \times$ that of DRAM.

The $\mathrm{RCD}$ receives addresses on the primary command-address bus (CAp), checks for cache hits/misses, and returns the status to the memory controller. This comparison logic plus address tag logic differentiate this RCD from the standard JEDEC RCD [9].

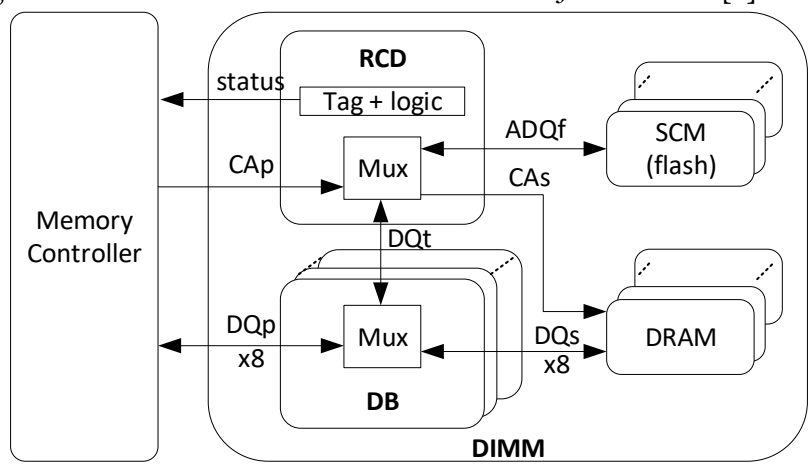

Figure 4: Half of hybrid DIMM with DRAM Cache and
SCM

A controller block on the RCD drives the command/data interface ADQf to the SCM and transfers blocks between SCM and DRAM. The DQt bus consists of a narrow, point-to-point connection between the RCD buffer and each of the ten $\mathrm{DB}$ buffers. With bidirectional data wires and a timing signal, it helps move blocks between DRAM and SCM. DQt buses differ from the $\mathrm{BCOM}$ bus in standard RCD/DB buffer chipsets. Steering logic in the RCD transfers data and addresses between CAp, CAs, ADQf, and DQt interfaces, while logic in the DBs transfers data between DQp, DQs, and DQt interfaces. The CAp and DQp buses and status are part of the Configuration D MEM bus in Fig. 1.

\subsection{L4 DRAM cache operation}

The RCD determines whether an incoming physical address hits or misses in the DRAM and communicates this status to the memory controller, which helps determine whether the returned read data is valid or the transaction must be retried after an interval. Likewise, the status tells it whether the write will finish immediately or be held in a write buffer for the L4 cache miss interval. In this paper we use the term L4 to refer to the physical DRAM cache. The RCD adjusts the address on the CAs to read data from or write data to the correct DRAM location. The 64B LLC line is sent over the DQs and DQp buses to the memory controller on a read or written from the controller on a write. The memory controller uses standard DRAM timings on the CAp and DQp buses. It chooses appropriate latency settings at initialization, e.g., to allow for tag comparison.

On a DRAM cache miss, the RCD adjusts the physical address to access the correct location in SCM. This address is driven on the ADQf bus to begin the slow access. Read data blocks are transported back to the Mux steering logic on the ADQf bus to be 
written to the DRAM via the DQt buses. The requested L3 line is returned first to minimize transport delay. Once the requested data is in DRAM, The memory controller retries the RD or WR transaction, which now hits in the L4.

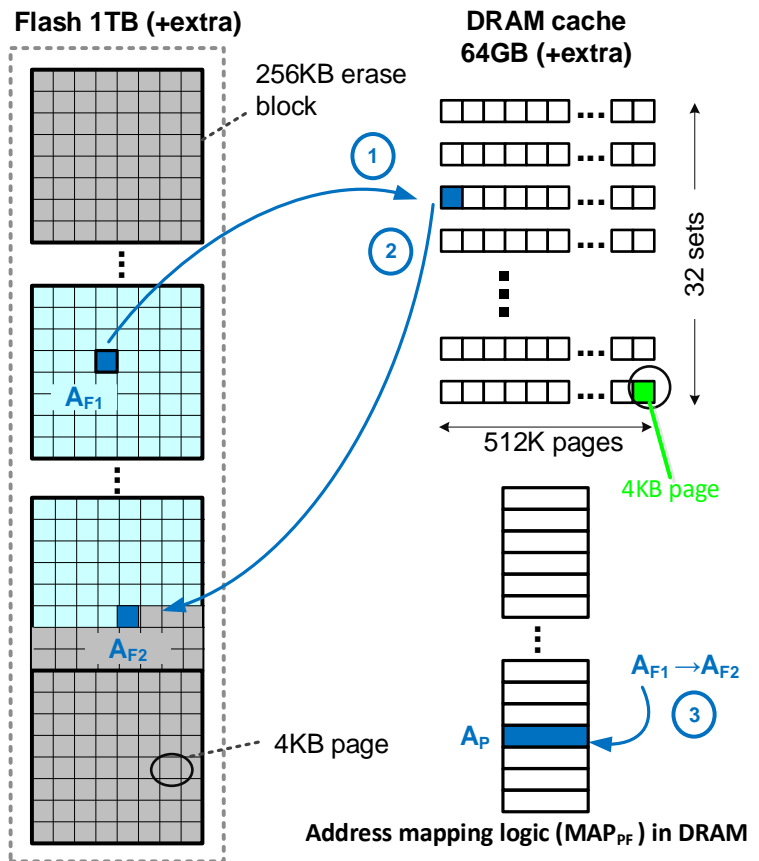

Figure 5: Physical-to-Flash address mapping

Fig. 5 shows the mapping logic to translate a physical page address to a Flash page address, assuming a 4KB L4 line size that is set to be identical to the Flash page size for simplicity. Other sizes can be supported with additional logic. The full mapping table only occupies about $0.1 \%$ of the Flash and about $1 \%$ of the DRAM.

A cache miss causes the page at address $A_{F 1}$ in Flash to be copied to a line in an L4 DRAM set (step 1). The L4 victim line is evicted (step 2) and, if dirty, is written into a new Flash page. This page, whose address is $\mathrm{AF}$, points to a $256 \mathrm{~KB}$ erase block that was previously erased and programmed. AF1's page is invalidated by modifying its entry in the DRAM mapping table to point to $A_{F 2}$ (step 3). The table is indexed by the physical address (AP in the figure) from the $\mathrm{RD} / \mathrm{WR}$ operation. AF1's entry in the reverse mapping table (not shown) is invalidated.

Without these mapping tables, evicting an L4 cache line would require erasing and reprogramming the entire erase block containing $A_{F 1}$. This would increase the Flash core activity by $64 \times$, reducing performance and causing unnecessary writes. Mapping largely eliminates the impact of erase-block granularity on performance and lifetime.

In order to minimize collisions during L4 cache evictions, we also added a hash function to select the Flash device that holds a DRAM address. When both write and a read have to be issued to Flash, the hash function minimizes the number of times the read and write go to the same Flash device.
Fig. 6 shows details of the DRAM cache. Here the L4 cache is organized as a 32-way set-associative cache with $4 \mathrm{~KB}$ L4 lines. Each set holds $512 \mathrm{~K}$ cache-lines or pages. Each $4 \mathrm{~KB}$ line holds 64 64-byte lines, where 64 bytes is both the LLC line size and the granularity for RDs and WRs. The stated line sizes refer to the data payload: the lines in both caches also contain $25 \%$ extra capacity.

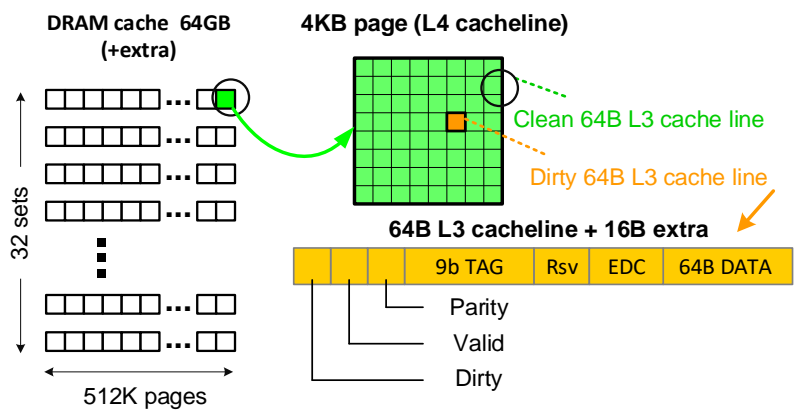

Figure 6: Tag details for L4 DRAM cache

Fig. 6 shows an LLC line with 64 data bytes, 8 bytes for error correction, three flag bits, nine address tag bits, and a 52-bit reserved field.

Every 64-byte LLC line within a given $4 \mathrm{~KB}$ L4 line is assigned the same nine-bit tag and valid flag when its data are transferred from Flash. The 64 dirty flags in an evicted L4 line must be scanned to determine if write-back is necessary.

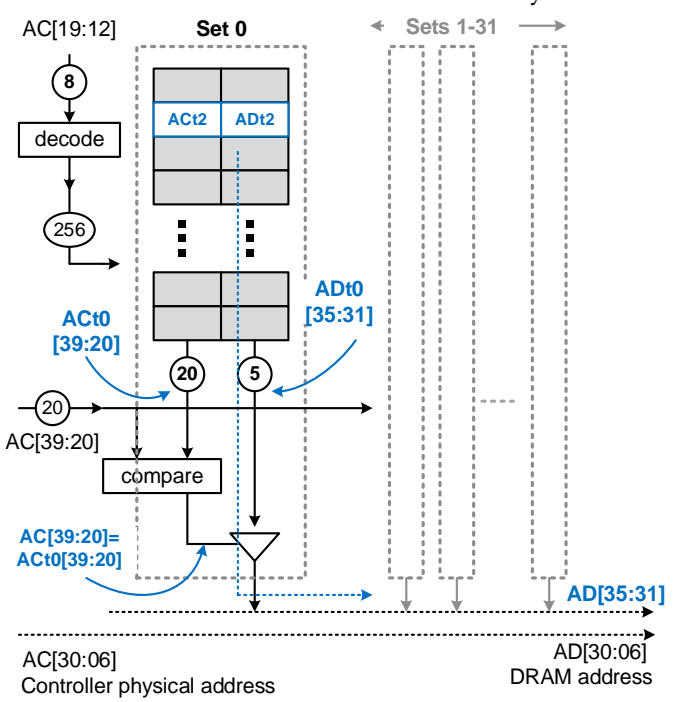

Figure 7: SRAM tag cache details

\subsection{SRAM tag cache details}

Fig. 7 shows details of an example 32-way set associative SRAM tag cache with 25-bit lines. Each set holds 256 lines. The resulting 8192 tags represent about $0.05 \%$ of the total needed for a 64 GB DRAM cache, which would require about $200 \mathrm{~kb}$ of SRAM 
within the RCD. In the figure, the $\mathrm{AC}$ [39:06] is the physical address produced by the memory controller for a $64 \mathrm{~B} \mathrm{~L} 3$ cacheline. The AC[39:12] field accesses the 32 tag cache sets in parallel. Each produces a 20-bit tag (ACtx[39:20]) that is compared to the corresponding AC[39:20] from the memory controller. On a match, the corresponding five-bit (ADtx[35:31]) field is used to form the DRAM address $\mathrm{AD}$ [35:06].

The time to service a miss to the tag cache is very short ( $40 \mathrm{~ns})$. The miss is handled by a sequence of pipelined reads to the appropriate tags of the L4 DRAM cache. Consequently, performance sensitivity to the tag cache size is low: reducing its size by $4 \times$ or $8 \times$ has little impact.

\section{EXPERIMENTAL METHODOLOGY}

We employ two simulation methodologies to model the behavior of our hybrid DIMM. The first is a simple model based on memory traces without timing information, and the second is a cycle-level, full-system simulator. The very slow latter model provides accurate performance measurements.

The simple model obtains traces of programs running on a real system and working with several GB of memory. We generate the traces by capturing all memory references using dynamic binary instrumentation tools (Valgrind [10] and Pin [11]) and modeling the cache hierarchy to generate the resulting memory activity that reaches the hybrid DIMM. We use these traces to study how the size, associativity, and line size impact the efficiency of the L4 DRAM cache.

The cycle-level model is based on the gem5 full-system simulator [12]. It simulates the relevant micro-architectural features of the device, allowing us to study the performance impact of parameter changes. Fig. 8 shows a block-level view of the internal components we implement in gem5.

Our cycle-level model simulates all functional and timing details that could impact performance, including DRAM commands, Flash operations, and ONFI bus [13] accesses. The model has been validated through extensive use of microbenchmarks.

Simulating full applications with this much slower model is infeasible. We therefore adopt Sherwood et al.'s SimPoint methodology [14] to select portions of an application that are most representative of the whole execution behavior. We simulate these parts to predict the final behavior. Our SimPoints consist of chunks of $100 \mathrm{M}$ instructions simulated after warm-up phases of $10 \mathrm{M}$ instructions. The limited number of instructions that we can simulate in a reasonable time frame limits the memory capacities of the systems that we can model, but our sampled simulation approach models the full system software stack.

\subsection{Workloads}

Since our hybrid DIMM targets data-center servers, we use six workloads from the CloudSuite benchmark collection [15]. These include: Data Serving, which is based on the NoSQL database; Apache Cassandra, which executes operations generated by the Yahoo! Cloud-Serving Benchmark (YCSB) [16]; Data Caching, which runs MemcacheD, a popular, distributed key-value, in- memory store [17]; Media Streaming, which uses the Nginx highperformance load balancer, web server, and content cache for streaming video content [18]; Graph Analytics, which tests the PageRank algorithm [19] over GraphX [20], a graph system for Apache Spark [21]; In-Memory Analytics, which runs the ALS algorithm provided by the Spark MLlib [22]; and Web Search, which uses the Apache Solr search engine framework [23].

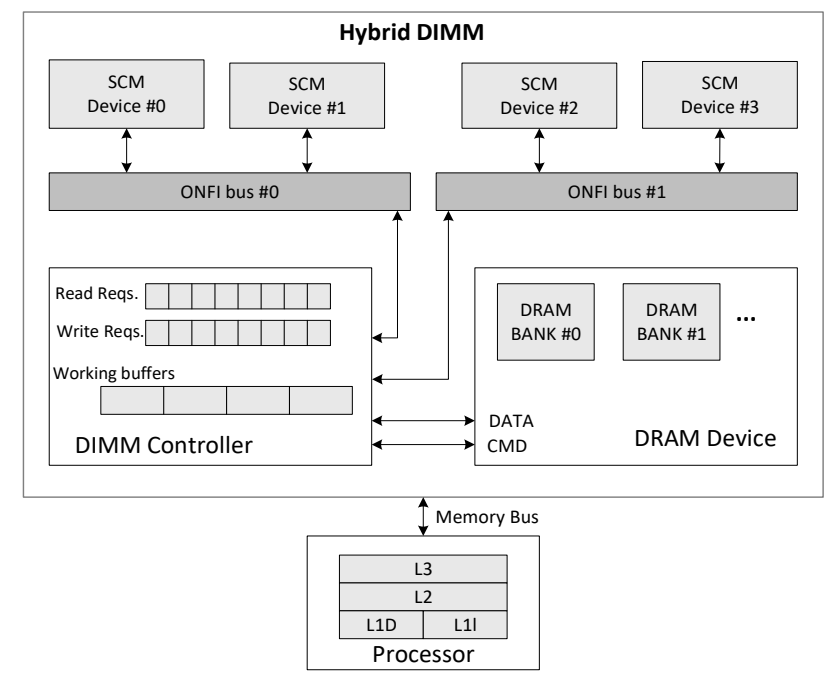

Figure 8: Simulation setup

\subsection{Experiments}

The goal of our experiments is predicting the performance of the Hybrid DIMM on a real system. We envision a system with a CPU like a Xeon ${ }^{\circledR}$ processor, with 32 cores and 6 Hybrid DIMMs, which results in a total amount of 6TB of SCM and 384GB of DRAM cache. The simulated CPU uses the following cache hierarchy: 32KB 8-way L1D (instruction and data); 256KB 8-way L2; and 32MB 16-way L3 cache.

Since this configuration is too big to be simulated by our tools and workloads, we have simulated a scaled-down version with $1 / 32$ the amount of resources. The resulting system has 1 CPU core and a Hybrid DIMM with 192GB of SCM and 12GB of DRAM cache. To properly scale-down the DRAM cache, its associativity needs to be reduced. This accounts for the conflicts that would occur on the real system, with more cores accessing the DRAM cache sets. Dividing the original associativity by 32 would result in a direct mapped cache, which is extreme. So we use a 4-way cache for our simulations as a better compromise.

We measure workload performance for different types of SCM, with each type being defined by its read and write latencies.

\section{RESULTS}

We compare performance, cost, endurance, and write behaviors on a range of SCM systems to those of a DRAM-only system for each of our data-center workloads.

Fig. 9 illustrates our hybrid DIMM performance for a range of $\mathrm{read} /$ write latencies relative to a baseline DRAM-only system that 
pessimistically assumes only $1 \mathrm{~TB}$ of physical memory. As shown in the first column of Table 3, the DRAM read and write latencies are 30ns; this time is measured from the row command to the delivery of the 64 bytes of column data.

For purposes of comparison, we define NewSCM to be a hybrid module built with a very low latency (300ns) SCM component, as shown in Table 3. It is byte-addressable and could potentially be built with any of the PCM, STT-MRAM, or ReRAM technologies in Table 1. We assume that the L4 cache for NewSCM is direct mapped and that tag and flag information are stored within the lines, as in Fig. 6.

The remaining hybrid systems use Flash components spanning a range of performance and cost points. Each configuration includes 1TB of Flash physical memory and 64GB of L4 DRAM cache. Read latencies range from $1-100 \mu \mathrm{s}$. Write latencies range from $100-660 \mu \mathrm{s}$. The block-addressable Flash requires an L4 line size of $4 \mathrm{~KB}$. Each line stores tag and flag information in its extra capacity, as in Fig. 6.

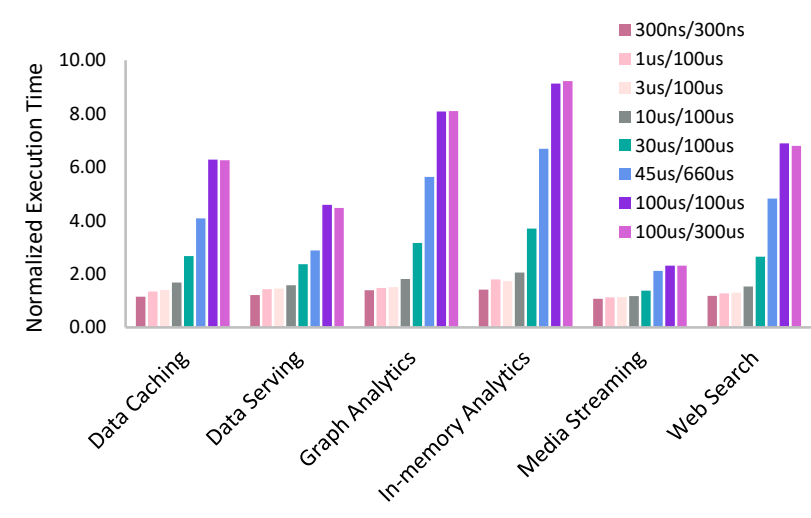

Figure 9: Relative execution time of hybrid versus DRAMonly memory systems for various Read/Write latencies of SCM (smaller is better)

We simulated a large set of applications, but in the interests of saving space, we present results for the six listed in Fig. 9. These six well represent the performances we observed. Since we estimate performance numbers using execution time, smaller numbers are better. For NewSCM, execution delays range from 7$42 \%$. Application delays are generally greater for Flash hybrid systems, ranging from slightly slower than NewSCM to about $6.5 \times$ slower when the read latency goes from $1 \mu$ s to $100 \mu$ s. Flash access latencies are one to two orders of magnitude worse than DRAM, but performance is only slightly worse for most workloads.

\subsection{Cost}

Table 3 illustrates relative costs per bit of main memories built from DRAM, NewSCM, and hybrid components using Flash of various latencies. We expect cost ratios between DRAM and SCM to continue to grow. We assume NewSCM costs $25 \%$ of DRAM and that the fastest Flash costs about $20 \%$. As discussed previously in the background section on Flash, Flash costs vary based on the features enabling different latencies (e.g., varying the bits stored per cell and number of cells on the word and bit lines).

Table 3: Relative costs of hybrid memory systems compared to a baseline 1TB DRAM-only memory system. SCM systems include 1TB SCM and 64GB DRAM L4 cache.

\begin{tabular}{l|lll|lll}
\hline \multirow{2}{*}{ Device } & \multicolumn{3}{|c|}{ Size } & \multicolumn{3}{c}{ Cost } \\
\cline { 2 - 7 } Read/Write latency & SCM & DRAM & Total & SCM & DRAM & Total \\
\hline All DRAM 30ns/ 30ns & 0 & 1 & 1 & 0 & 1 & 1 \\
NewSCM 300ns/ 300ns & 1 & 0.0625 & 1.0625 & 0.25 & 0.0625 & 5 \\
Flash 1us/100us & 1 & 0.0625 & 1.0625 & 0.19 & 0.0625 & 0.2525 \\
Flash 3us/100us & 1 & 0.0625 & 1.0625 & 0.17 & 0.0625 & 0.2325 \\
Flash 10us/100us & 1 & 0.0625 & 1.0625 & 0.088 & 0.0625 & 0.1505 \\
Flash 30us/100us & 1 & 0.0625 & 1.0625 & 3 & 0.0625 & 0.1255 \\
Flash 45us/660us & 1 & 0.0625 & 1.0625 & 0.06 & 0.0625 & 0.1225 \\
Flash 100us/100us & 1 & 0.0625 & 1.0625 & 0.057 & 0.0625 & 0.1195 \\
Flash 100us/300us & 1 & 0.0625 & 1.0625 & 0.045 & 0.0625 & 0.1075 \\
\hline
\end{tabular}

The rightmost column of Table 3 shows total memory system cost when using hybrid components. Each configuration provides at least 1TB physical memory. SCM configurations also include 64GB DRAM cache.

\subsection{Cost/performance}

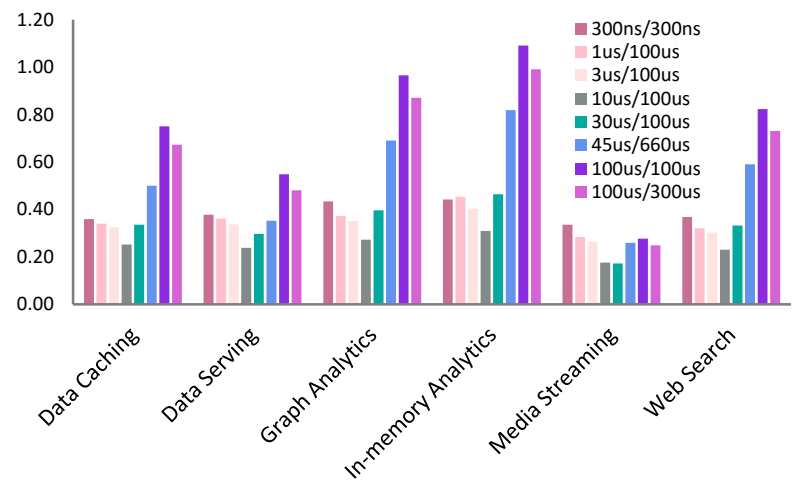

Figure 10: Cost/performance of NewSCM and hybrid Flash relative to a DRAM-only baseline for various $\mathrm{Read} / \mathrm{Write}$ latencies of SCM (smaller is better)

Fig. 10 illustrates relative cost per performance - execution time multiplied by memory system cost - for a range of read/write latencies compared to a DRAM-only system. The NewSCM system delivers a cost/performance value that is up to $3 \times$ better than the all-DRAM configuration. The hybrid Flash system with $10 \mu$ s read latency shows the best cost/performance benefits: up to $5.6 \times$ better than DRAM alone. The Flash configurations with read latencies of $100 \mu \mathrm{s}$ exhibit worse cost-performance than the NewSCM configuration for most workloads. 


\subsection{Flash SCM endurance}

Flash endurance is lowest of the SCM technologies in Table 1, so it is important to minimize the number of Flash writes. We measure write counts and use them to extrapolate the system lifetime for each application. Table 4 shows estimated endurance for different workloads. Although we simulate a single-core system, we calculate endurance assuming a system with 32 cores, $6 \mathrm{~GB}$ of L4 DRAM, and $16 \times$ SCM per core. The write bandwidth is set to the measured value on a real system, which is a pessimistic estimation, since the workloads would run slower on the real system. Target endurance is $32.8 \mathrm{~PB}$ writes per TB of SCM. All cores run the same application to better exercise the entire $1 \mathrm{~TB}$ memory. A background process implements wear leveling to distribute writes across the Flash blocks.

With only one exception, the system lifetimes exceed the U.S. SEC's three-year nominal depreciation interval for capital equipment. The outlier, Graph Analytics, reduces system lifetime to about six months. Its high write count likely poses an issue for other SCM technologies, as well.

The L4 DRAM cache control logic tries to maximize Flash lifetime. It prioritizes clean L4 lines to evict when the number of dirty L4 lines within the Flash page is sufficiently low. Otherwise it prioritizes the writeback. The threshold could be userconfigurable or managed via a hardware monitor. The controller could also vary the amount of cache allocated to each process to try to provide enough capacity to hold the application's working set and thus absorb most of the 64B write transactions in the DRAM. Outlier applications like Graph Analytics need specific run-time parameters to guarantee that they receive a greater L4 capacity in order to absorb more writes. The system will likely include some regular DRAM components to which applications with little locality and high write-to-read ratios can be allocated. Putting processes to sleep can also relieve pressure on the L4 cache.

\subsection{Flash write behaviors}

An evicted L4 line gets written into a previously erased and programmed Flash page. A 4KB L4 cache line contains 64 LLC lines, some of which may be dirty. Since Flash is a block device, the entire $4 \mathrm{~KB}$ must be written, causing unnecessary writes for the clean LLC lines. We define write amplification to be the ratio of total bytes to the number of dirty bytes written per page. Table 4 shows these values for our workloads. The average of about 2.8 (ignoring Media-Streaming) implies that only $~ 35 \%$ of the LLC lines are dirty within each L4 line. Unlike Flash, the Table 1 SCM components that support byte-granularity accesses avoid this.

When a $4 \mathrm{~KB}$ L4 line is evicted, some of its dirty LLC lines may have been written more than once. This is beneficial: the DRAM cache absorbs write operations without impacting SCM lifetime. We define write absorption to be the average number of writes that occur before eviction for each dirty 64B LLC line. The write absorption counts in Table 4 average to about 2.3 writes before eviction. We again ignore Media Streaming, which is designed to have a very small memory footprint and hence difficult to force to use more memory. In addition, the application serves only static data, so it issues few writes, making it impossible to compute the write absorption and write amplification metrics for this workload. Nonetheless, these same properties mean that system endurance definitely exceeds three years.

Table 4: Hybrid DIMM Flash lifetimes

\begin{tabular}{lllll}
\hline Workload & $\begin{array}{l}\text { Time } \\
\text { (Years) }\end{array}$ & $\begin{array}{l}\text { Cost } \\
\text { Factor }\end{array}$ & $\begin{array}{l}\text { Write } \\
\text { Amp. }\end{array}$ & $\begin{array}{l}\text { Write } \\
\text { Abs. }\end{array}$ \\
\hline Data Caching & 9.02 & 1 & 4.70 & 1.23 \\
Data Serving & 7.37 & 1 & 5.81 & 2.61 \\
Graph Analytics & 0.44 & 6.85 & 1.07 & 2.95 \\
In-Memory & & & & \\
Analytics & 5.71 & 1 & 1.38 & 2.66 \\
Media Streaming & infinite & 1 & infinite & infinite \\
Web Search & 93.86 & 1 & 1.21 & 2.12 \\
\hline
\end{tabular}

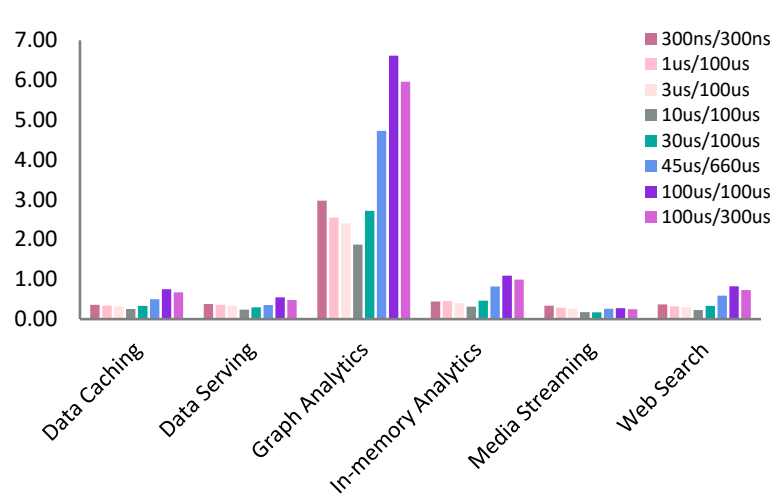

Figure 11: Cost/performance with endurance factored in for NewSCM and hybrid Flash relative to a DRAM-only baseline for various Read/Write latencies of SCM (smaller is better)

\section{ANALYSIS AND DISCUSSION}

Here we analyze performance sensitivity to different design parameters: access latencies, operation interruptibility, and L4 DRAM cache configuration. We generate different views of our data to better understand our empirical results and to project the full-system impact of adopting devices such as our hybrid DIMM.

\subsection{Read access time}

Fig. 12 presents another perspective of read-latency impact on application execution time for a fixed write latency of 100us. The data used for this view are the same as used to generate Fig. 9. The figure illustrates the near-linear dependence of execution time upon SCM read latencies. When a load misses in the on-chip cache hierarchy there is usually a limited number of additional instructions that can be executed before the core reaches an instruction that depends on the SCM data. 


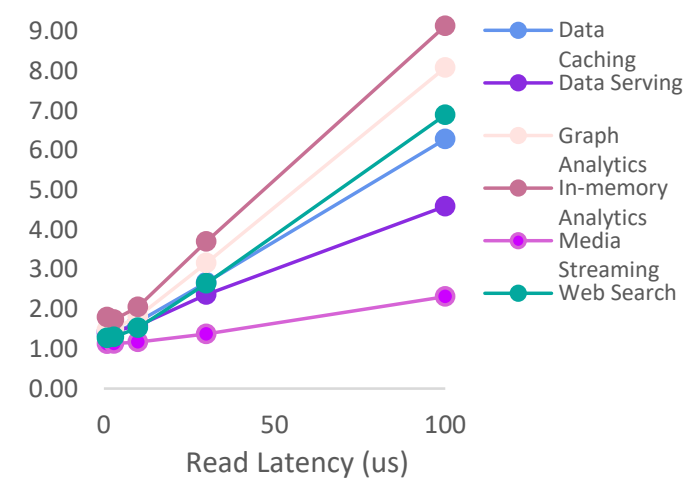

Figure 12: Normalized execution time relative to Flash read latency for a fixed write latency of 100us for various workloads (smaller is better)

The execution time $\mathrm{T}_{\mathrm{i}}$ of an application can be expressed as $\mathrm{T}_{\mathrm{i}}$ $\sim\left(\mathrm{K}_{\mathrm{i}}{ }^{\text {onchip }}+\mathrm{tRD}^{\mathrm{H}} \times \mathrm{K}_{\mathrm{i}}{ }^{4 \mathrm{H}}\right)+\mathrm{t} \mathrm{RD}-\mathrm{M} \times \mathrm{K}_{\mathrm{i}}{ }^{4 \mathrm{M}}$. $\mathrm{K}_{\mathrm{i}}{ }^{\text {onchip }}$ refers to accesses hitting in the on-chip L1/L2/L3 cache hierarchy, $\mathrm{t}_{\mathrm{RD}}-\mathrm{H}^{\times} \mathrm{K}_{\mathrm{i}}{ }^{4 \mathrm{H}}$ refers to accesses hitting in the L4 DRAM cache, and $t_{R D}-M^{\times} \times \mathrm{K}_{\mathrm{i}}{ }^{4 \mathrm{M}}$ refers to accesses missing in the on-DIMM L4. The access time of L4 hit is tRD-H, and that of miss is (requiring an SCM access) is tRD-M. The $y$-intercept in Fig. 12 is defined by $\mathrm{K}_{\mathrm{i}}^{\text {onchip }}+\mathrm{tRD}-\mathrm{H} \times \mathrm{K}_{\mathrm{i}}{ }^{4 \mathrm{H}}$, where $\mathrm{K}_{\mathrm{i}}{ }^{4 \mathrm{M}}$ is the slope.

The near-linear dependence is partly a consequence of the limitations of the simulation environment. We simulate a single core executing one application. Multi-threading within the executing application and context switches to OS are included, but context switches to another application are not included. We scale the shared LLC, the L4 DRAM cache, and the SCM physical memory down to appropriate sizes for single applications on a single core. A real system would include many cores executing different applications. These applications would interfere with each other in the shared LLC, the L4 DRAM cache, and the SCM physical memory. This is a topic for future investigation.

\subsection{Write access time}

Write latency does not directly impact execution time because LLC writes from the memory controller are held in data/address buffers in the RCD and DB components shown in Fig. 4. On an L4 hit the data are written immediately, but on a miss they must await an SCM access. For subsequent read accesses to the write location, the RCD address-comparison logic and buffering makes it appear as if the write transaction completed with the L4 hit latency.

The parameters of the resources that handle L4 write misses could affect execution time. These include the number of writemiss buffers in the RCD/DBs, the ADQf bus bandwidth, and the Flash write bandwidth. If there is not enough room for SRAM buffers on the DIMM, space in the L4 DRAM cache could be set aside for buffering purposes.

We do not expect the bandwidth of the ADQf bus not to be a significant factor. We assume the ADQf buses on the module are both 16 bits wide and operate at $800 \mathrm{Mb} / \mathrm{s}$. This results in an aggregate bandwidth of $3.2 \mathrm{~GB} / \mathrm{s}$. A $4 \mathrm{~KB}$ L4 line has a transport time of about $2.5 \mathrm{us}$ per bus. With a $100 \mu$ s Flash write access time and 64 Flash components on the module, 64 concurrent writes take $80 \mu \mathrm{s}$ transport time on the two ADQf buses. Flash write bandwidth becomes a limiting factor if the write access time is greater than $80 \mu \mathrm{s}$. However, the $100 \mu \mathrm{s} / 100 \mu \mathrm{s}$ and $100 \mu \mathrm{s} / 300 \mu \mathrm{s}$ $\mathrm{read} /$ write times for Flash have essentially the same application execution times. We therefore conclude that the number of Flash writes is low enough that we are not yet hitting resource limitations. This is also a topic for further investigation.

\subsection{Operation interruptibility}

Even though execution time is not directly affected by SCM write access time, latency-sensitive Flash reads can be delayed by long writes. We avoid this by adding a write-interrupt feature to the hybrid DIMM.

A Flash write operation sets the erased memory cells of a page to a programmed state and then reads them back to verify that the program threshold has been met. The process repeats for memory cells that are still below the threshold. This operation can be interrupted between each iterative step, allowing a higher priority read of a different Flash page to proceed. The interrupted write continues after the read. If the high-priority read is to the page being written, the data are supplied by a write buffer on the RCD and DB components. Note that the relatively short transport time of an L4 line ( 2.5 $\mu \mathrm{s})$ means that ADQf bus interference impacts effective read latency much less.

The Flash erase-block operation resets the memory cells to an erased state and then reads the data back to see if the erase threshold has been met. As with writes, the process repeats for cells that are still below the threshold. This operation can be interrupted in between each iterative step, allowing a highpriority read to a different Flash page to proceed. The interrupted erase then continues. Recall that all data in an erase block have been moved to other pages, invalidating the pages in the erase block. This means there can never be a high-priority read directed to the erase block.

Making Flash reads interruptible would also permit higher priority reads to proceed ahead of lower priority ones. These interrupt features require modifying the sequencing logic of the Flash component to stop an operation at the proper step and adding commands to handle the different cases to the ADQf interface logic.

Fig. 13 shows how much interruptible Flash writes reduce execution time compared to identical systems without this feature (Fig. 9). Performance for most workloads improves significantly, particularly for SCM components whose writes are long relative to the reads. The cases were the performance is worse are caused by differences in the code simulated. When write latency is high, it takes longer to simulate a series of instructions, leading to a higher probability that the instruction sequence changes due to factors such as operating system timer, different scheduling decisions depending on the progress of processes etc. 


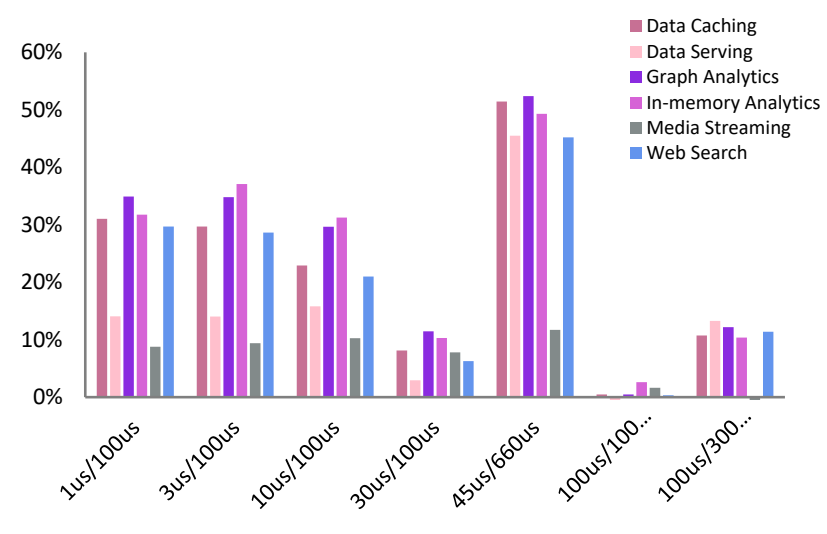

Figure 13: Relative performance improvement of hybrid DIMM systems for various Read/Write latencies of Flash when Flash writes are interruptible (higher is better)

\subsection{L4 line size}

We initially experimented with 64-byte L4 lines to match those of the LLC. Two things became quickly apparent. First, there is very little data reuse in the L 4 cache; reuse mostly occurs in the L1/L2/L3 caches. Second, there is a lot of spatial locality within $4 \mathrm{~KB}$ regions of physical memory: when one LLC line is accessed within an L4 line, well over half the remaining 64B LLC lines get accessed as well. This is because data within a given physical page tend to be closely related, whereas data in the next physical page are probably unrelated due to the way the OS maps a contiguous virtual address space to a fragmented physical address space.

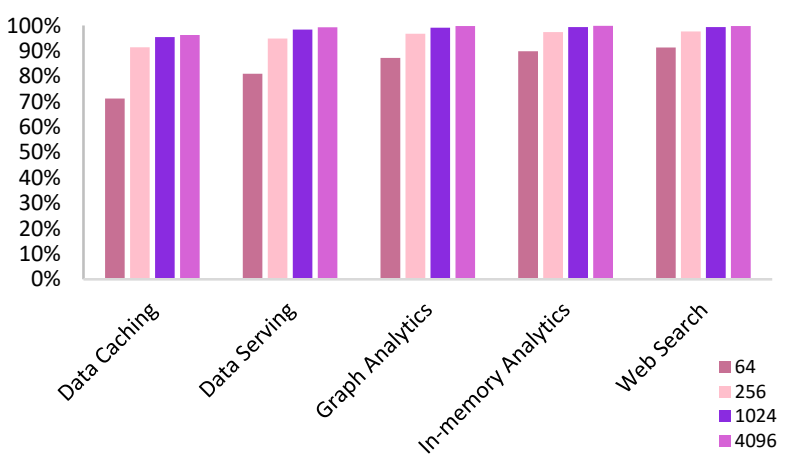

Figure 14: Cache hit ratio for different L4 line sizes (higher is better) $4 \mathrm{~KB}$ DRAM cache lines give the best hit ratio due to the spatial locality

Fig. 14 illustrates the $\mathrm{L} 4$ cache hit ratio for our range of memory module configurations using L4 line sizes of $64-4 \mathrm{~K}$ bytes.
Fig. 15 shows the corresponding impact on performance. $4 \mathrm{~KB}$ gives the highest cache hit ratio and correspondingly the lowest execution time. Prefectching $4 \mathrm{~KB}$ data from the Flash allows us to take advantage of the spatial locality in most workloads resulting in increased hit ratio. 4KB L4 DRAM cache line matches the Flash page size and the virtual-to-physical translation granularity, which also simplifies our design. For other Flash page sizes, we would still use the virtual-to-physical mapping size for the L4 lines, letting addressing logic manage the mismatch.

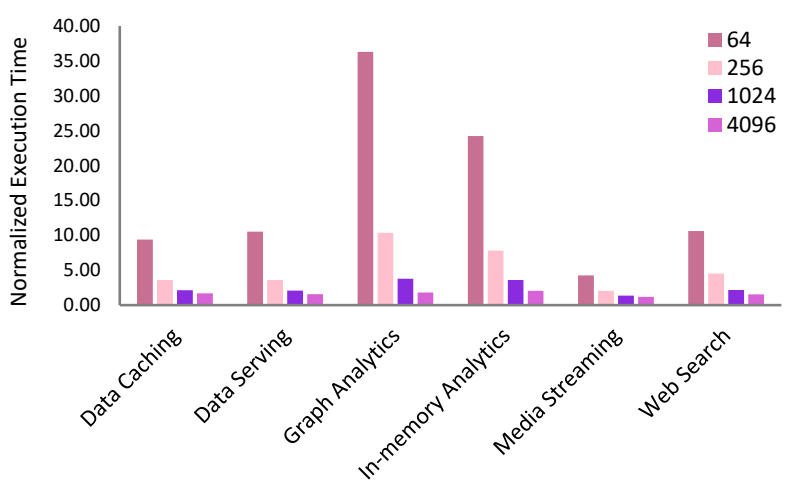

Figure 15: Performance sensitivity to L4 cache line size (smaller is better). $4 \mathrm{~KB}$ is better due to the improved hit ratio

\subsection{L4 associativity and tag storage}

Our simulations confirm that a direct-mapped L4 cache cannot hold dirty L4 lines long enough to absorb Flash writes. Most of our studies use 32-way L4 caches, giving us flexibility in exploring different eviction policies. This associativity is also large enough that there are still enough sets to avoid a high conflict miss rate when memory resources are scaled down for single core execution.

Storing L4 cache-line tags in a small SRAM in the RCD makes cache-controller sequencing easy. Other storage options include external SRAM and tags stored within the L4 DRAM lines themselves. Implementation considerations lead us to choose the SRAM tag organization discussed in Section 4.2. All storage methods perform similarly.

\subsection{L4 capacity}

The L4 DRAM cache size is 64GB for the data payload, which uses two DDP $16 \mathrm{~Gb}$ DRAMs at each of 20 DRAM sites. This represents the maximum size that is easily realizable. The minimum size is $16 \mathrm{~GB}$, assuming one $8 \mathrm{~Gb}$ DRAM at each DRAM site. Performance differences across this range of capacity are fairly small. Fig. 16 confirms that the performance sensitivity to various L4 to SCM capacity ratios is low. A key concern is the impact of L4 capacity on system lifetime. A smaller L4 may not absorb Flash writes well: L4 capacity misses can cause dirty L4 cache-lines to be written to Flash more often than they would with a larger L4. 


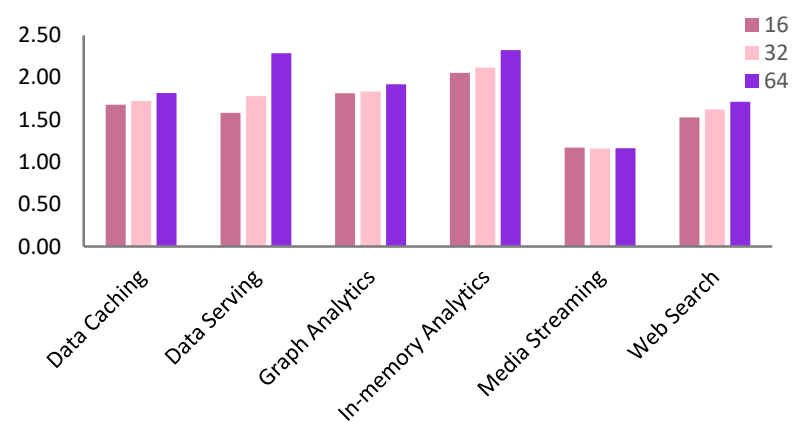

Figure 16: Relative execution time of a hybrid SCM system with 10us/100us latency to a DRAM-only system for various L4 to SCM capacity ratios (smaller is better)

\subsection{L4 write policy}

Our L4 DRAM cache policy is write-back/write-allocate. Write-back caches may contain both clean and dirty lines, whereas write-through caches only hold clean lines because updated lines are immediately written out to main memory. Write-through caches cannot absorb writes, which lowers system lifetime. Write-allocate caches bring data into cache on a write miss, possibly displacing resident data. We need this policy to help minimize write amplification.

\subsection{L4 eviction policy}

The 32-way L4 associativity creates several issues with respect to set management. The first is the fact that storing the tags for the 64 LLC lines within an L4 line requires extra capacity. This is most cleanly handled with an SRAM tag cache. The distributed tags for the 32 ways are accessed relatively infrequently, and so the penalty for this is low. A second issue is the eviction policy: the simplest way to choose a set from which to evict a line is to employ pseudo-random selection, since no history need be tracked. Adopting 32-way associativity spreads victimization well.

Our pseudo-random selection considers the clean/dirty status of the L4 lines. The cache controller prefers to evict clean lines unless the clean/dirty ratio has dropped below a statically assigned threshold. This keeps write absorption high while maintaining good read performance.

\section{RELATED WORK}

We briefly survey other Flash-based devices and SCM mainmemory systems. Note that most of the systems referenced here are academic proposals that have been simulated or emulated. In contrast, we plan to prototype the hybrid memory system described in this paper.

\subsection{DIMM Devices}

Micron Technology's recent NVDIMM-Ns back DRAM with Flash into a single memory subsystem that supports DRAM-like latencies and persistence [24]. Unlike the hybrid memory solution proposed in this paper, the role of Flash on NVDIMM-N is not to add capacity at low cost, but rather as a backup during power failure. Diablo Technologies Memory1 ${ }^{\mathrm{TM}}$ [25] [26] also increases memory capacity by placing Flash in a DDR4 DIMM that uses standard memory slots to house up to four terabytes of Memory1 at a fraction of the cost of the highest-capacity DRAMs. Diablo heterogeneous main memory uses software to manage data between the DRAM and Memory1 tiers, providing capacity, performance, and endurance while requiring no changes to application or system software. This is different from the hardware management of DRAM cache discussed in this paper. Smart Storage Solutions [27] also attaches a DIMM containing conventional Flash directly to the memory bus, making it visible to the OS and allowing it to be accessed either via a block device driver or a memory extension driver. In HybriDIMM solution by Samsung and Netlist [28], Flash can only be accessed from the host as a block device. This is different from the 64B access size supported on the host memory bus for the entire SCM address range in this paper.

All these components are managed by software, whereas our proposed hybrid DIMM is entirely managed in hardware. The NVDIMM and Memory1 devices target persistent memory systems, whereas our hybrid DIMM targets data-center server memory systems.

\subsection{Flash as Main Memory}

Grupp et al. [29] strive to understand trade-offs between Flash performance, cost, and reliability together with how different usage patterns affect these characteristics. They find that Flash manufacturers provide conservative guidelines about these metrics, and this lack of detail makes it difficult to design systems that fully exploit Flash capabilities. They empirically characterize devices from five vendors, finding that performance varies significantly among vendors and devices. In particular, they find their measurements vary significantly from publicly available datasheets. They also demonstrate and quantify some unexpected device characteristics and show how they can be used to improve responsiveness and energy consumption. They target SSDs, but their findings are relevant for Flash main memory.

The idea of using direct-attached conventional Flash as main memory is not new. Wu and Zwaenopol [30] first proposed a large main memory built primarily with Flash over two decades ago. Their eNVy system maps an array of persistent storage whose controller sits on the memory bus. Their simulated system models copy-on-write page remapping, a small amount of SRAM, and high bandwidth parallel data transfers at latencies that support 30,000 transactions per second on the TPC-A database benchmark.

Each processor in Caulfield et al.'s Gordon system [31] has access to a high bandwidth, DDR-attached Flash array. They propose several FTL modifications in their Flash controller. For instance, they find that increasing the number of write points and spreading accesses among them increases dynamic parallelism and thus write bandwidth. Striping data to create super-pages increases effective bandwidth and throughput, and merging incoming and pending read requests and combining write requests to the same pages makes the super-page granularity work well for large transfer sizes. 
Sudan et al. [32] study a hybrid main memory in which 24 GB of DRAM functions as a cache for the slower 160GB of Flash main memory, and the tiering between these technologies is handled transparently to the application. They find that their system delivers a bit less than a third of the TPC-C performance of a DRAM-only system at a small fraction of the cost.

\subsection{Other Technologies as Main Memory}

Lee et al. [33] first study PCM as a main memory alternative due to its similar energy and performance and its amenability to scaling, proposing architectural modifications to improve performance and endurance.

Qureshi et al. [34] couple up to 32GB of PCM with an 8GB DRAM buffer. These are backed by Flash and then a hard disk. The buffer is comprised of normal DIMMs and organized as a hardware cache. It is managed by the DRAM controller and invisible to the OS. Like us, they find that the DRAM buffer filters most SCM writes. They also propose simple organizational and management solutions that reduce PCM write traffic, increasing endurance three-fold.

Tavana et al. [35] seek to improve PCM reliability to the point where it can tolerate many hardware faults. They replace faulty bits with bits normally used for metadata, relying on read verification and an extra write operation to detect/locate faults in the main storage array.

García et al. [36] also seek to increase the endurance of PCM or ReRAM used as a DRAM replacement. They study the combination of last-level cache replacement policies, compression, and wear leveling. The first two reduce the number of writes, increasing lifetime by an order of magnitude, whereas careful wear leveling can increase lifetime by two orders of magnitude.

Zhao et al. [37] propose Kiln, a persistent memory design that adopts a nonvolatile cache and a nonvolatile main memory to enable atomic in-place updates without logging or copy-on-write. This direct-attached memory system composed of byteaddressable non-volatile technologies such as PCM or STTMRAM strives deliver performance very close to that of a system without persistence support.

Jung and Cho [38] also target persistence. They propose Memorage, a system architecture that integrates management of all physical resources for memory and storage. Memorage uses the OS's virtual memory manager to improve the performance of memory-intensive workloads and extend main memory lifetime. Their prototype Linux implementation with 8 GB persistent RAM main memory and 240- 480 GB persistent RAM storage increases system lifetime by factors of about four to seven.

Like most of these memory subsystems, our hybrid memory architecture can accommodate other byte-addressable SCMs such as PCM, ReRAM, and STT-MRAM paired with a DRAM cache. Furthermore, our architecture enables the pairing of low-cost, block-level enhanced Flash components with a DRAM cache.

\subsection{Stacked DRAM Cache}

Alloy Cache [39] by Qureshi and Loh, ATCache [40] by Huang and Nagarajan and Unison Cache [41] by Jevdjic et al. discuss the use of high bandwidth, low latency, stacked DRAM as a cache for slower offchip DRAM. In this case, the read/write latency difference between the two DRAM types is not as significant as that between DRAM and enhanced Flash or SCM, so the architectural considerations are different.We find that set associative cache that improves hit rate performs better than direct mapped cache. Accessing the tags for the 32 ways in a single access avoids multiple serial accesses for the tags. Unlike Alloy cache, we still use separate DRAM accesses for tag and data. A small SRAM tag cache, similar to the approach in ATCache, reduces the hit latency by avoiding the need for DRAM tag access in majority of the cases. We use $4 \mathrm{~K}$ page-based DRAM cache as opposed to the 64B block based DRAM cache used by ATCache since this reduces the tag storage overhead in DRAM for the large capacities associated with SCM. Both Alloy Cache and Unison Cache avoid SRAM tag storage and rely instead on MissMap predictor and way predictor respectively to improve performance.

\section{CONCLUSIONS AND FUTURE WORK}

As DRAM capacity scaling slows, using SCM to augment or replace DRAM in future computer systems becomes increasingly attractive. Flash now has - and is likely to maintain - the lowest cost per bit. Unfortunately, Flash has many issues relative to other SCM technologies - higher access latency, lower component bandwidth, larger access granularity, smaller endurance values, and non-deterministic access delays. We show that these disadvantages can be alleviated by mapping Flash to the physical address space on the memory bus and using an L4 DRAM cache in front of the SCM. Our simulations show that L4 sizes of 1664GB have very high hit rates, letting the processor enjoy DRAM latencies/bandwidths for most accesses. Handling the infrequent cache misses requires that the processor's memory controller be modified to be able to retry transactions.

Adapting the L4 DRAM line size to the Flash page size prefetches contiguous LLC cache lines into the DRAM cache, which takes advantage of the spatial locality present in most applications. The L4 DRAM cache's large associativity combined with an eviction policy that prioritizes victimizing clean lines increases write absorption to improve memory lifetime. In addition, our write-amplification results show that blockaddressed Flash fronted by a large DRAM cache functions well: the endurance of block-oriented SCM rivals those of byteaddressed components for many workloads.

The bottom line is that our hybrid module with enhanced Flash and an L4 DRAM cache performs well for many data-center workloads. On average, a system using our hybrid DIMM with enhanced Flash having a $3 \mu$ s read latency achieves up to $88 \%$ of the performance of an all-DRAM system with 30ns access times. Our system exhibits up to $94 \%$ the performance of a 300ns SCM system employing a similar DRAM cache. When factoring in cost, our hybrid module has even better value - up to $5.2 \times$ better than an all-DRAM memory system and up to $1.4 \times$ better than a $300 \mathrm{~ns}-$ access-time SCM with a similar L4 DRAM cache.

We have begun studying the impact of simultaneously executing different applications on multiple cores, and we are exploring the ramifications of architectural design choices on 
power, persistence, and reliability. We are also investigating the impact of electrical and thermal noise, in general, and the impact of write latency for smaller read latencies. Our current plan is to prototype Configuration D.

\section{REFERENCES}

[1] S.-H. Lee, "Technology Scaling Challenges and Opportunities of Memory Devices," in International Electron Devices Meeting, 2016.

[2] D. Kang, W. Jeong, C. Kim, D.-H. Kim, Y. S. Cho, K.-T. Kang, K. Ryu, K.-M. Kang, S. Lee, W. Kim, H. Lee, J. Yu, N. Choi, D.-S. Jnag, C. A. Lee, Y.-S. Min, M.-S. Kim, A.-S. Park, J.-I. Son, I.-M. Kim, P. Kwak, B.-K. Jung, J.-Y. Lee, K.-T. Park and K.-H. Kyung, "256 Gb 3 b/Cell V-NAND Flash Memory with 48 Stacked WL Layers," IEEE Journal of Solid-State Circuits, vol. 52, no. 1, 2017.

[3] C. Kim, J.-H. Cho, W. Jeong, I.-h. Park, H.-W. Park, D.-H. Kim, D. Kang, S. Lee, J.-S. Lee, W. Kim, J. Park, Y.-l. Ahn, J. Lee, J.-h. Lee, S. Kim, H.-J. Yun, J. Yu, N. Choi, Y. Kwon, N. Kim, H. Jang, J. Park, S. Song, Y. Park, J. Bang, S. Hong, B. Jeong, H.-J. Kim, C. Lee, Y.-S. Min, I. Lee, I.-M. Kim, S.-H. Kim, D. Yoon, K.-S. Kim, Y. Choi, M. Kim, K. Huunggon, P. Kwak, J.-D. Ihm, D.-S. Byeon, J.-y. Lee, K.-T. Park and K.-h. Kyung, "A 512Gb 3b/cell 64-Stacked WL 3D V-NAND Flash," in Proc. IEEE International Solid State Circuits Conference, 2017.

[4] B. Tallis, "Samsung at Flash Memory Summit: 96-Layer V-NAND, MLC Z-NAND, New Interfaces," AnandTech, 9 August 2017.

[5] J. Choe, "SK hynix' 21 nm DRAM Cell Technology: Comparison of 1st and 2nd generation," Tech Insights, 5 June 2017. [Online]. Available: http://techinsights.com/abouttechinsights/overview/blog/sk-hynix-21-nm-dram-celltechnology-comparison-of-1st-and-2nd-generation/. [Accessed 12 November 2017].

[6] Wooseong Gheong, et al., Samsung Electronics, "A Flash Memory Controller for 15us Ultra-Low-Latency SSD Using HighSpeed 3D NAND Flash with 3us Read Time," in IEEE International Solid-State Circuits Conference, 2018.

[7] Z. Cui, T. Lu, S. A. McKee, M. Chen, H. Pan and Y. Ruan, "TwinLoad: Bridging the Gap between Conventional Direct-Attached and Buffer-on-Board Memory Systems," in Proc. International Conference on Memory Systems, 2016.

[8] J. Chang and A. Sainio, "NVDIMM-N Cookbook: A Soup-to-Nuts Primer on Using NVDIMM-Ns to Improve Your Storage," Storage Networking Industry Association, 2015.

[9] JEDEC, "DDR4 Registering Clock Driver (DDR4RCD01)," 2016.

[10] N. Nethercote and J. Seward, "Valgrind: a framework for heavyweight dynamic binary instrumentation," in Proc. ACM SIGPLAN Conference on Programming Language Design and Implementation, 2007.

[11] C.-K. Luk, R. Cohn, R. Muth, H. PAtil, A. Klauser, G. Lowney, S. Wallace, V. J. Reddi and K. Hazelwood, "Pin: Building Customized Program Analysis Tools with Dynamic
Instrumentation," in Proc. ACM SIGPLAN Conference on Programming Language Design and Implementation, 2005.

[12] N. Binkert, B. Beckmann, G. Black, S. K. Reinhardt, A. Saidi, A. Basu, J. Hestness, D. R. Hower, T. Krishna, S. Sardashti, R. Sen, K. Sewell, M. Shoaib, N. Vaish, M. D. Hill and D. A. Wood, "The gem5 simulator," ACM SIGARCH Computer Architecture News, vol. 39, no. 2, pp. 1-7, 2011.

[13] ONFI Working Group, "Open NAND Flash Interface Specification Revision 3.0,"

www.onfi.org/ /media/onfi/specs/onfi_3_0_gold.pdf?la=en, 2011.

[14] T. Sherwood, E. Perelman, G. Hamerly and B. Calder, "Automatically Characterizing Large Scale Program Behavior," in Proc. ACM International Conference on Architectural Support for Programming Languages and Operating Systems, 2002.

[15] M. Ferdman, A. Adileh, O. Kocberber, S. Volos, M. Alisafaee, D. Jevdjic, C. Kaynak, A. D. Popescu, A. Ailamaki and B. Falsafi, "Clearing the Clouds: A Study of Emerging Scale-out Workloads on Modern Hardware," in Proc. International Conference on Architectural Support for Programming Languages and Operating Systems, 2012.

[16] B. F. Cooper, A. Silberstein, E. Tam, R. Ramakrishnan and R. Sears, "Benchmarking Cloud Serving Systems with YCSB," in Proc. ACM Symposium on Cloud Computing, 2010.

[17] B. Fitzpatrick, "Distributed Caching with Memcached," Linux Journal, no. 124, p. 5, 2004.

[18] W. Reese, "Nginx: the High-Performance Web Server and Reverse Proxy," Linux fournal, no. 173, p. 2, 2008.

[19] L. Page, S. Brin, R. Motwani and T. Winograd, "The PageRank Citation Ranking: Bringing Order to the Web," 1999.

[20] R. S. Xin, J. E. Gonzalez, M. J. Franklin and I. Stoica, "GraphX: A Resilient Distributed Graph System on Spark," in International Workshop on Graph Data Management Experiences and Systems, 2013.

[21] M. Zaharia, R. S. Xin, P. Wendell, T. Das, M. Armbrust, A. Dave, X. Meng, J. Rosen, S. Venkataraman, M. J. Franklin, A. Ghodsi, J. Gonzalez, S. Shenker and I. Stoica, "Apache Spark: A Unified Engine for Big Data Processing," Communications of the ACM, vol. 59, no. 11, pp. 56-65, 2016.

[22] X. Meng, J. Bradley, B. Yavuz, E. Sparks, S. Venkataraman, D. Liu, J. Freeman, D. B. Tsai, M. Amde, S. Owen, D. Xin, R. Xin, M. J. Franklin, R. Zadeh, M. Zaharia and A. Talwalker, "MLlib: Machine Learning in Apache Spark," Journal of Machine Learning Research, vol. 17, pp. 1235-1241, 2016.

[23] D. Smiley, E. Pugh, K. Parisa and M. Mitchell, Apache Solr Enterprise Search Server, 3 ed., Packt Publishing Ltd, 2015.

[24] Micron Technology, Inc., NVDIMM: Persistent Memory Performance, 2016.

[25] Diablo Technologies, "Memory1 Solution Whitepaper," May 2016. [Online]. Available: https://www.diablo- 
technologies.com/wp-content/uploads/2016/05/Memory1Whitepaper.pdf. [Accessed 17 October 2017].

[26] M. L. Takefman, M. Amer and R. Badalone, "System and method of interfacing co-processors and input/output devices via a main memory system". USA Patent US8713379, 29 April 2014.

[27] J. Scaramuzzo, "Reaching the Final Latency Frontier," in Keynote at Flash Memory Summit, 2013.

[28] Netlist, "HYBRIDIMM ${ }^{\mathrm{TM}}$ : Storage at Memory Speeds, Memory at Storage Capacities.," [Online]. Available:

http://www.netlist.com/products/Storage-Class-

Memory/HybriDIMM/default.aspx. [Accessed 257 2018].

[29] L. M. Grupp, A. M. Caulfield, J. Coburn, S. Swanson, E. Yaakobi, P. H. Siegel and J. K. Wolf, "Characterizing Flash Memory: Anomalies, Observations, and Applications," in Proc. IEEE/ACM International Symposium on Microarchitecture, 2009.

[30] M. Wu and W. Zwaenepoel, "eNVy: a Non-Volatile, Main Memory Storage System," in Proc. ACM International Conference on Architectural Support for Programming Languages and Operating Systems, 1994.

[31] A. M. Caulfield, L. M. Grupp and S. Swanson, "Gordon: Using Flash Memory to Build Fast, Power-efficient Clusters for Dataintensive Applications," in Proc. ACM International Conference on Architectural Support for Programming Languages and Operating Systems, 2009.

[32] K. Sudan, A. Badam and D. Nellans, "NAND-Flash: Fast Storage or Slow Memory?," in Non-Volatile Memory Workshop, 2012.

[33] B. C. Lee, E. Ipek, O. Mutlu and D. Burger, "Architecting Phase Change Memory as a Scalable DRAM Alternative," in Proc. ACM/IEEE International Symposium on Computer Architecture, 2009 .

[34] M. K. Qureshi, V. Srinivasan and J. A. Rivers, "Scalable High Performance Main Memory System Using Phase-Change Memory Technology," in Proc. ACM/IEEE International Symposium on Computer Architecture, 2009.

[35] M. Khavari Tavana, A. K. Ziabari, M. Arjomand, M. Kandemir, C. Das and D. Kaeli, "REMAP: A Reliability/Endurance Mechanism for Advancing PCM," in Proc. International Symposium on Memory Systems, 2017.

[36] A. A. García, W. Wang, R. de Jong and S. Diestelhorst, "Composing Lifetime-Enhancing Techniques for Non-Volatile Main Memories," in Proc. International Conference on Memory Systems, 2017.

[37] J. Zhao, S. Li, D. H. Yoon, Y. Xie and N. P. Jouppi, "Kiln: Closing the Performance Gap Between Systems," in Proc. IEEE/ACM International Symposium on Microarchitecture, 2013.

[38] J.-Y. Jung and S. Cho, "Memorage: Emerging Persistent RAM Based Malleable Main Memory and Storage Architecture," in Proc. ACM International Conference on Supercomputing, 2013.

[39] M. K. Qureshi and G. H. Loh, "Fundamental Latency Trade-offs in Architecting DRAM Caches: Outperforming Impractical
SRAM-Tags with a Simple and Practical Design," in Proc. IEEE/ACM International Symposium on Microarchitecture, 2012.

[40] C.-C. Huang and V. Nagarajan, "ATCache: Reducing DRAM Cache Latency via a Small SRAM Tag Cache," in Proc. International Conference on Parallel Architectures and Compilation Techniques, 2014.

[41] D. Jevdjic, G. H. Loh, C. Kaynak and B. Falsafi, "Unison Cache: A Scalable and Effective Die-Stacked DRAM Cache," in Proc. IEEE/ACM International Symposium on Microarchitecture, 2014. 